\title{
Microenvironmental VEGF concentration, not total dose, determines a threshold between normal and aberrant angiogenesis
}

\author{
Clare R. Ozawa, ${ }^{1}$ Andrea Banfi, ${ }^{1}$ Nicole L. Glazer, ${ }^{2}$ Gavin Thurston, ${ }^{3}$ \\ Matthew L. Springer, ${ }^{1}$ Peggy E. Kraft, ${ }^{1}$ Donald M. McDonald, ${ }^{2}$ \\ and Helen M. Blau ${ }^{1}$ \\ ${ }^{1}$ Baxter Laboratory in Genetic Pharmacology, Stanford University School of Medicine, Stanford, California, USA \\ ${ }^{2}$ Cardiovascular Research Institute, Comprehensive Cancer Center, and Department of Anatomy, University of California, \\ San Francisco, California, USA \\ ${ }^{3}$ Regeneron Pharmaceuticals Inc., Tarrytown, New York, USA
}

\begin{abstract}
Use of long-term constitutive expression of VEGF for therapeutic angiogenesis may be limited by the growth of abnormal blood vessels and hemangiomas. We investigated the relationship between VEGF dosage and the morphology and function of newly formed blood vessels by implanting retrovirally transduced myoblasts that constitutively express $\mathrm{VEGF}_{164}$ into muscles of adult mice. Reducing VEGF dosage by decreasing the total number of VEGF myoblasts implanted did not prevent vascular abnormalities. However, when clonal populations of myoblasts homogeneously expressing different levels of VEGF were implanted, a threshold between normal and aberrant angiogenesis was found. Clonal myoblasts that expressed low to medium levels of VEGF induced growth of stable, pericyte-coated capillaries of uniform size that were not leaky and became VEGF independent, as shown by treatment with the potent VEGF blocker VEGF-TrapR1R2. In contrast, clones that expressed high levels of VEGF induced hemangiomas. Remarkably, when different clonal populations were mixed, even a small proportion of cells with high production of VEGF was sufficient to cause hemangioma growth. These results show for the first time to our knowledge that the key determinant of whether VEGF-induced angiogenesis is normal or aberrant is the microenvironmental amount of growth factor secreted, rather than the overall dose. Long-term continuous delivery of VEGF, when maintained below a threshold microenvironmental level, can lead to normal angiogenesis without other exogenous growth factors.
\end{abstract}

J. Clin. Invest. 113:516-527 (2004). doi:10.1172/JCI200418420.

\section{Introduction}

VEGF induces vascular growth in physiological and pathological settings of adult angiogenesis (1-4). Because of its potency, VEGF delivery is being tested in clinical trials for its ability to promote therapeutic angiogenesis in adult ischemic heart and limb muscles. Results obtained to date appear promising (5-10). However, the vessels induced by VEGF frequently display morphological and functional abnormalities that can call into question the therapeutic utility of VEGF delivery. For example, exogenous VEGF administration

Received for publication March 21, 2003, and accepted in revised form December 16, 2003.

Address correspondence to: Helen M. Blau, Baxter Laboratory in Genetic Pharmacology, Stanford University School of Medicine, Stanford, California 94305-5175, USA. Phone: (650) 723-6209; Fax: (650) 736-0080; E-mail: hblau@stanford.edu.

Matthew L. Springer's present address is: Division of Cardiology, University of California, San Francisco, California, USA.

Clare R. Ozawa and Andrea Banfi contributed equally to this work.

Conflict of interest: Gavin Thurston is an employee of Regeneron Pharmaceuticals Inc.

Nonstandard abbreviations used: platelet-endothelial cell

adhesion molecule 1 (PECAM-1); $\alpha$-smooth muscle actin $(\alpha$-SMA). during embryonic vasculogenesis (11) or VEGF overexpression in various tissues in transgenic animals $(12-14)$ results in malformed leaky vessels with unusually large irregular lumens. VEGF gene delivery to both ischemic and nonischemic tissues, using such diverse delivery methods as myoblast-mediated gene transfer $(15,16)$, injection of naked plasmid DNA $(5,17)$ and adenoviral vectors $(18,19)$, causes growth of tumor-like vessels and hemangiomas.

The physiological angiogenic response requires a precisely coordinated interplay of different signaling molecules and cell types. This has led to the investigation of strategies for the codelivery or coupregulation of other factors together with VEGF for therapeutic angiogenesis (for an overview, see ref. 20). On the other hand, genetic studies have shown that even small variations in levels of VEGF expression have profound effects on vascular development, as embryonic lethality results from as little as a $50 \%$ reduction $(21,22)$ or a two- to threefold increase (23) in VEGF expression.

In this study, we sought to understand the relationship between the dosage of delivered VEGF and the induction of normal or pathological angiogenesis in adult muscle. Furthermore, as VEGF $_{164}$ is 
known to bind and to be stored in the extracellular matrix, we investigated whether normal angiogenesis can be achieved by manipulating total VEGF expression or if microenvironmental levels must instead be carefully controlled to avoid overdosage. Local delivery of VEGF was achieved by implantation of VEGF $_{164}$-expressing myoblasts into the adult mouse ear, a thin and accessible organ with muscle that is amenable to whole-mount analysis of morphological and functional changes in its vascular network (14, $24,25)$. The effects of VEGF delivery were examined in nonischemic tissue to avoid the confounding hypoxia/hypoglycemia-induced expression of multiple angiogenic factors and therefore to minimize the effects of factors other than VEGF on the resulting angiogenic response.

We alternatively controlled the overall dose of VEGF or the maximal microenvironmental level of VEGF secretion by implanting (a) different proportions of a heterogeneous population of VEGF-expressing myoblasts or (b) clonal populations of myoblasts that homogeneously produced VEGF at different levels. We found that, indeed, VEGF induces normal angiogenesis or aberrant vessels and hemangiomas in a strictly dose-dependent fashion. However, the key factor was the microenvironmental level of VEGF secretion, not the overall dose. Moreover, a discrete threshold in dosage separating normal and pathological angiogenesis was revealed. These results demonstrate for the first time to our knowledge that continuous expression of VEGF at the appropriate dose can produce normal and stable vascular growth in the absence of additional exogenous factors. The findings underscore the need for the development of gene therapy protocols that control microenvironmental release, not just overall dose, to exploit the therapeutic potential of VEGF while avoiding aberrant vasculature.

\section{Methods}

Cell culture. Primary myoblasts isolated from C57BL/6 mice and transduced to express the $\beta$-galactosidase marker gene (LacZ) from a retroviral promoter (26) were further infected at high efficiency with four successive rounds of MFG-VEGF 164 retrovirus $(15,27)$. For the creation of VEGF* myoblasts, a second population of LacZ-expressing C57BL/6 myoblasts was infected with a retrovirus similar to the MFG-VEGF ${ }_{164}$ retrovirus, except sequences encoding a hemagglutinin epitope tag (28) (YPYDVPDYA) were engineered at the $3^{\prime}$ end of the VEGF CDNA by PCR.

Early-passage myoblast clones were isolated using a FACStar cell sorter (Becton Dickinson, Franklin Lakes, New Jersey, USA), and single-cell isolation was confirmed visually. The stability of the VEGF secretion was assessed periodically by ELISA on aliquots of three clones across the VEGF production spectrum. VEGF production was within $10 \%$ of reference levels, demonstrating that the clones reproducibly secrete predictable amounts of VEGF. Myoblasts were cultured in $5 \% \mathrm{CO}_{2}$ on collagen-coated dishes as previously described $(26,27)$.

Implantation of myoblasts into mice. Male SCID CB.17 mice 6-8 weeks old (Taconic, Germantown, New York, USA) were treated in accordance with institutional guidelines, after approval from the Stanford University ethical committee. SCID mice were used to avoid an immunological response to $\beta$-galactosidase-expressing myoblasts (data not shown). Myoblasts were dissociated in trypsin and resuspended in PBS with $0.5 \%$ BSA. Myoblasts $\left(5 \times 10^{5}\right.$ in $\left.5 \mu \mathrm{l}\right)$ were implanted into the posterior auricular muscle, midway up the dorsal aspect of the external ear, using a syringe with a 29.5-gauge needle.

VEGF ELISA measurements. Cell culture supernatants and mouse sera were quantified for VEGF protein using an ELISA kit (R\&D Systems, Minneapolis, Minnesota, USA). For assessment of VEGF production by cells in culture, $1 \mathrm{ml}$ of medium was harvested from myoblasts in one $60-\mathrm{mm}$ dish after a 4-hour incubation, filtered, and analyzed in duplicate. Results were normalized for the number of cells and time of exposure to medium. Three dishes of cells were assayed per cell type. Mouse serum for ELISA measurements was obtained from blood samples that were allowed to clot at room temperature for several hours and were centrifuged twice.

Tissue staining. The entire vascular network of the ear could be visualized after intravascular staining with a biotinylated Lycopersicon esculentum lectin that binds the luminal surface of all blood vessels, as previously described $(14,24,25)$. Mice were anesthetized and lectin was injected intravenously, and 2 minutes later the tissues were fixed by vascular perfusion of $1 \%$ paraformaldehyde and $0.5 \%$ glutaraldehyde in PBS, $\mathrm{pH}$ 7.4. Ears were then removed, bisected in the plane of the cartilage, and stained with X-gal staining buffer $(1 \mathrm{mg} / \mathrm{ml}$ 5-bromo-4-chloro-3-indoyl- $\beta$-D-galactoside, $5 \mathrm{mM}$ potassium ferricyanide, $5 \mathrm{mM}$ potassium ferrocyanide, $0.02 \%$ Nonidet P- $40,0.01 \%$ sodium deoxycholate, $1 \mathrm{mM} \mathrm{MgCl}_{2}$, in PBS, $\mathrm{pH}$ 7.4). Tissues were stained using avidin-biotin complex-diaminobenzidine histochemistry (Vector Laboratories, Burlingame, California, USA), dehydrated through an alcohol series, cleared with toluene, and whole-mounted on glass slides with Permount embedding medium (Fisher Scientific, Fair Lawn, New Jersey, USA) $(14,24)$. Vascular morphology was analyzed at 18 hours, 4 days, and 1,2 , and 4 weeks, and at various time points between 1.5 and 3.5 months after myoblast implantation for specific experiments.

For tissue sections, mice were anesthetized and sacrificed by cervical dislocation. The ears were harvested, embedded in OCT compound (Sakura Finetek, Torrance, California, USA), frozen in isopentane cooled in liquid nitrogen, and cryosectioned. Tissue sections were then stained with X-gal (20- $\mu \mathrm{m}$ sections) or with $\mathrm{H} \& \mathrm{E}(10-\mu \mathrm{m}$ sections) as described previously $(26,29)$. Immunofluorescence was performed essentially as previously 
described (15). The following primary antibodies and dilutions were used: rat anti-mouse platelet endothelial cell adhesion molecule-1 (PECAM-1; Pharmingen, San Diego, California, USA) at a dilution of 1:100; mouse anti-mouse $\alpha$-smooth muscle actin ( $\alpha$-SMA; ICN Biomedicals, Aurora, Ohio, USA) at a dilution of 1:400; rabbit anti-NG2 (Chemicon International, Temecula, California, USA) at a dilution of 1:200; and goat anti-collagen type IV (Chemicon International) at a dilution of 1:200. Fluorescence-labeled secondary antibodies (Molecular Probes, Eugene, Oregon, USA) were used at a dilution of 1:100.

Plasma leakage measurements. Evans blue assays were performed as previously described $(14,25)$. Evans blue (J.T. Baker, Phillipsburg, New Jersey, USA) was injected into the femoral vein at a dose of 30 micrograms per gram of mouse body weight. Four hours later, mice were perfused with $1 \%$ paraformaldehyde in 0.05 $\mathrm{M}$ citric acid, $\mathrm{pH}$ 3.5. Full-thickness biopsy punches of ears with a diameter of $6 \mathrm{~mm}$ (Sklar Instruments, West Chester, Pennsylvania, USA) were obtained and weighed. Evans blue was extracted from tissue with formamide at $55^{\circ} \mathrm{C}$ overnight and was measured with a spectrophotometer at $610 \mathrm{~nm}$. Amounts are expressed as nanograms of dye per milligram tissue wet weight. Plasma leakage was measured 4 days and 4 weeks after myoblast implantation.

VEGF-Trap R1R2. VEGF-Trap ${ }_{\text {R1R2, }}$, which consists of portions of the extracellular domains of VEGFR-1 and VEGFR-2 coupled to human Fc, is a soluble form of VEGF receptors that can be used to deplete active VEGF in vivo (30). VEGF-Trapr1R2 was used to test the dependence of the vascular structures induced by different VEGF levels on continued VEGF production. VEGF myoblast clones or LacZ myoblasts (controls) were implanted in the ears of mice. Mice were treated with VEGF-TrapR1R2 $(25 \mathrm{mg} / \mathrm{kg} ; 100 \mu \mathrm{l}$ intraperitoneally) in PBS (40 $\mathrm{mM}$ phosphate and $20 \mathrm{mM} \mathrm{NaCl}$, $\mathrm{pH}$ 7.4) or with vehicle (PBS; $100 \mu \mathrm{l}$ intraperitoneally) 2 and 4 days before harvest. On days 7 and 28, the vasculature was stained by injection of biotinylated $L$. esculentum lectin i.v. to examine the morphological changes ( $n=4-8$ mice per group, except for LacZ myoblasts: $n=3$ mice).

Vessel measurements. For the creation of tracings of areas of effect, regions containing bulbous vascular structures or capillary proliferation with approximately $50 \%$ or greater vessel length densities over controls (see arrows in Figure 1f for examples) were traced onto clear plastic overhead projector sheets from captured and live video microscopic images. Areas of effect were then measured from these tracings using a digitizing tablet. Vessel diameters and length densities were measured in whole mounts of ears stained with L. esculentum lectin. Vessel diameters were measured by overlay of a live video microscopic image with a computer-generated square lattice. Squares were chosen at random and the diameter of
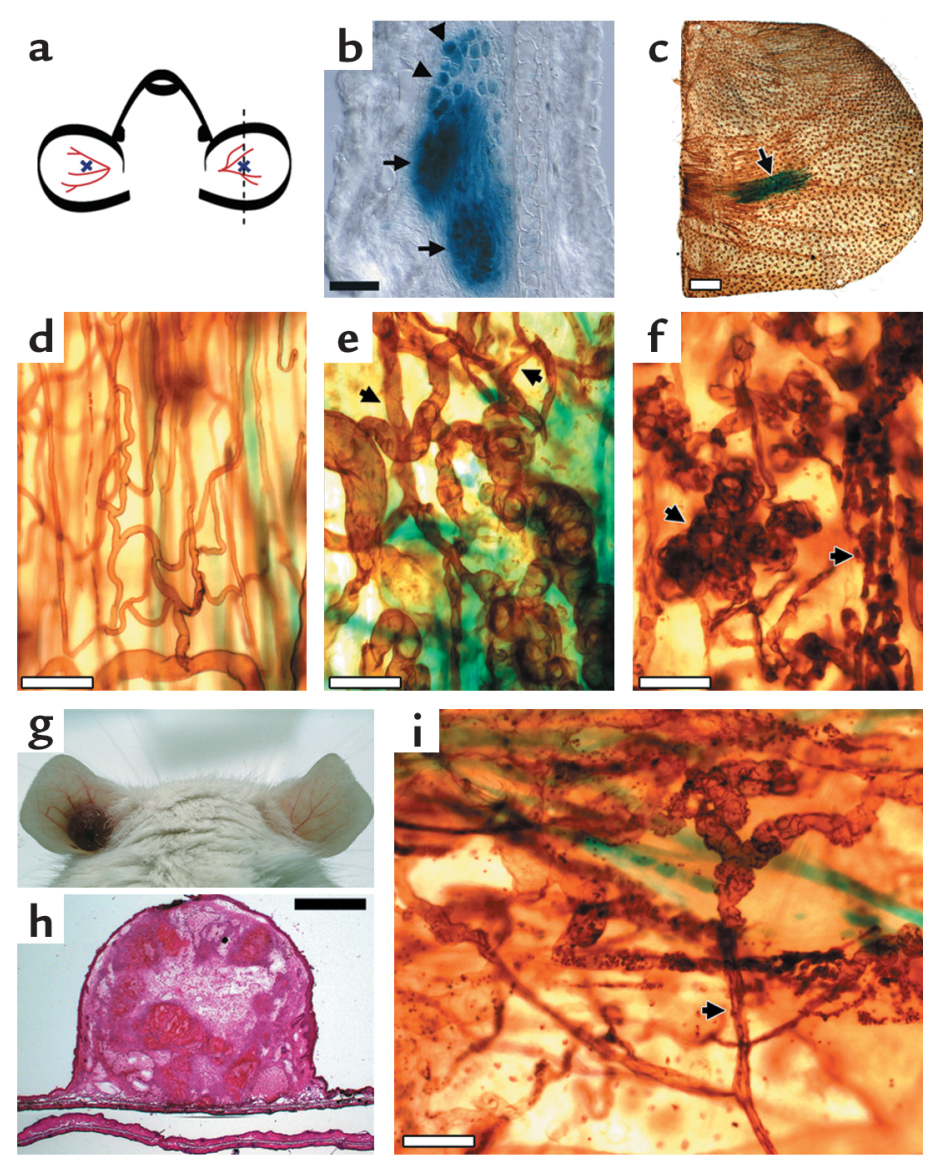

\section{Figure 1}

Uncontrolled VEGF production promotes abnormal vascular growth in muscle. (a) Myoblasts expressing both $\mathrm{VEGF}_{164}$ and LacZ or control LacZ myoblasts were implanted into the posterior auricular muscles of mice (crossmarks). (b) X-gal staining of a control ear sectioned along the dashed line in a at 14 days after implantation, showing LacZ myoblasts fused into endogenous myofibers (arrowheads) and clusters of unfused myoblasts or myoblasts fused with each other (arrows; $n=7$ ). (c) Lectin and X-gal staining of control ear bisected in the plane of the cartilage, showing LacZ-positive myofibers at the site of injection at 28 days (arrow; $n=10$ ). (d) Vessels at sites of implanted control myoblasts were normal at all time points (18 hours and 4, 7, 14, 28, and 64 days; $n=10$ for each time point). (e) At 7 days after implantation of VEGF myoblasts, vessels leading into the injection site (arrows) became enlarged $(n=9)$. (f) At 28 days after implantation, aberrant bulbous structures (left arrow) and bundles of capillary-like vessels (right arrow) were present at sites of implanted VEGF myoblasts $(n=10)$. (g) At day 64, typical large blood-filled growths (hemangiomas) developed in ears implanted with VEGF myoblasts (left). The contralateral control ear was normal $(n=10)$. (h) H\&E staining of an ear containing a hemangioma (top) and of a control ear (bottom). (i) A vessel leading into the injection site (arrow) became abnormal in the immediate vicinity of implanted VEGF myoblasts (from a second VEGF myoblast population, VEGF*; 7 days after implantation). Scale bars: $100 \mu \mathrm{m}(\mathbf{b}), 1 \mathrm{~mm}(\mathbf{c}$ and $\mathbf{h})$, and $50 \mu \mathrm{m}(\mathbf{d}-\mathbf{f}$ and $\mathbf{i})$. 
each vessel (where present) in the center of selected squares was measured. Thirty vessel diameter measurements were obtained per ear, and three ears were analyzed per group $(n=3)$. Vessel length density was measured as previously described (25) on three fields per ear and three ears per group $(n=3)$.

Statistics. Data are presented as means \pm standard error. Correlation coefficients were calculated using linear regression analysis. The significance of differences between vessel diameter measurements was assessed using the Kolmogorov-Smirnoff two-sample test. $P<0.05$ was considered statistically significant (Statview 4.5 software; Abacus Concepts, Berkeley, California, USA).

\section{Results}

Uncontrolled VEGF production induces growth of abnormal blood vessels. For expression of genes in adult muscle tissue, primary myoblasts were transduced with a constitutive LacZ-encoding retrovirus alone or together with a murine $\mathrm{VEGF}_{164}$-encoding retrovirus and were implanted into the thin posterior auricular muscle of the adult mouse external ear (Figure 1a).
To first confirm that myoblasts delivered to the ear fuse with endogenous myofibers and provide a localized source of transgene expression, we injected myoblasts transduced with LacZ retrovirus alone into the posterior auricular muscle and examined them at various time points by X-gal staining. Implanted myoblasts elongated and fused with each other or with surrounding host myofibers by 14 days after implantation (Figure 1b). At day 28, injected myoblasts were fully fused at the implantation site and continued to produce $\beta$-galactosidase (Figure 1c). Myoblasts remained localized at the site of injection at all time points.

We examined blood vessels induced by implanting a polyclonal population of myoblasts containing heterogeneous retroviral integration sites and producing VEGF at diverse levels (VEGF myoblasts). Vessels in control ears implanted with LacZ-expressing myoblasts (control myoblasts) remained unchanged at all time points examined (from 18 hours to 64 days), and were primarily capillaries oriented parallel to auricular muscle fibers (Figure 1d). In contrast, vessels surrounding VEGF myoblasts underwent a

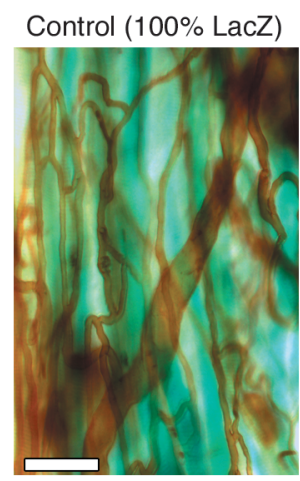

b
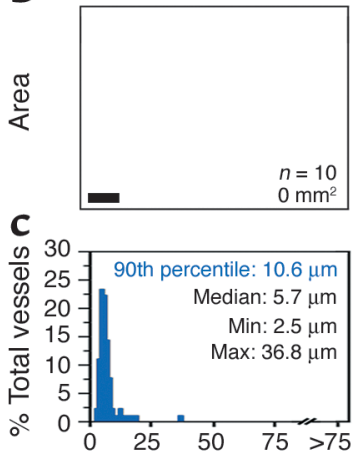
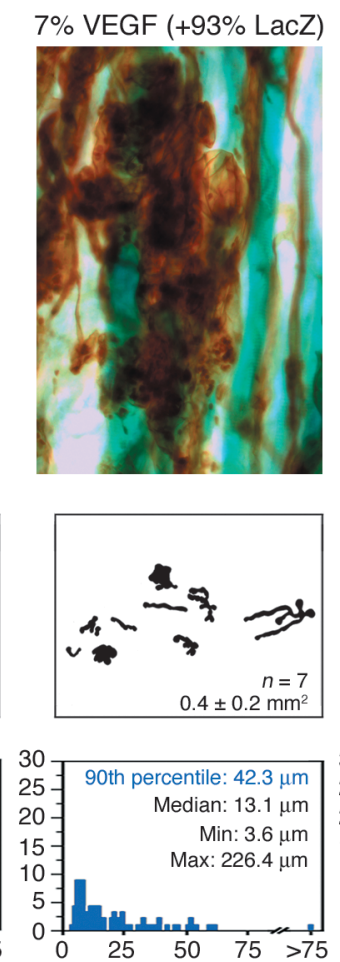
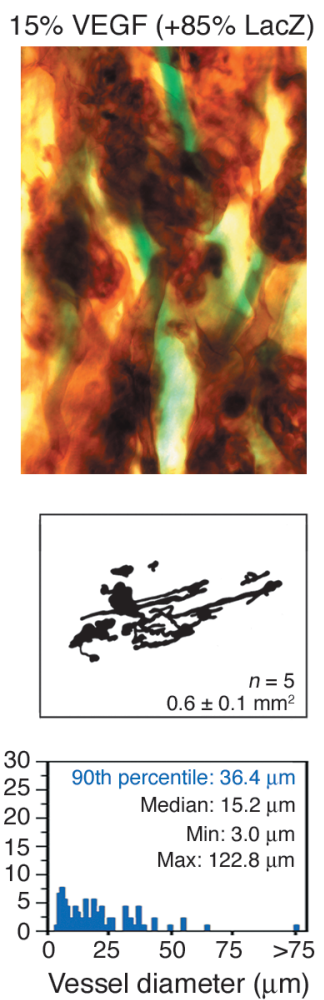
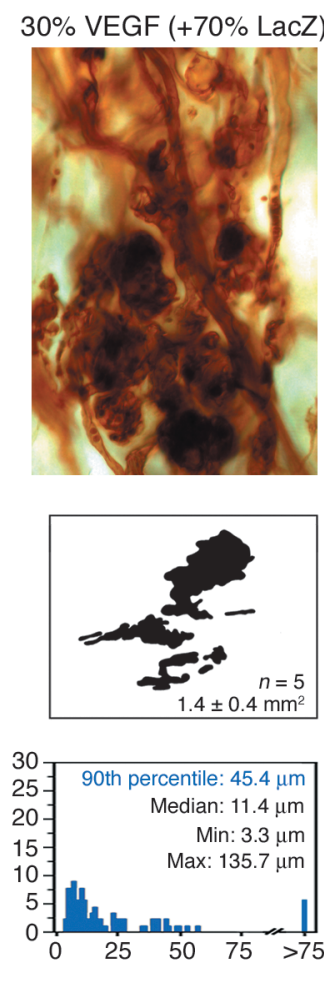
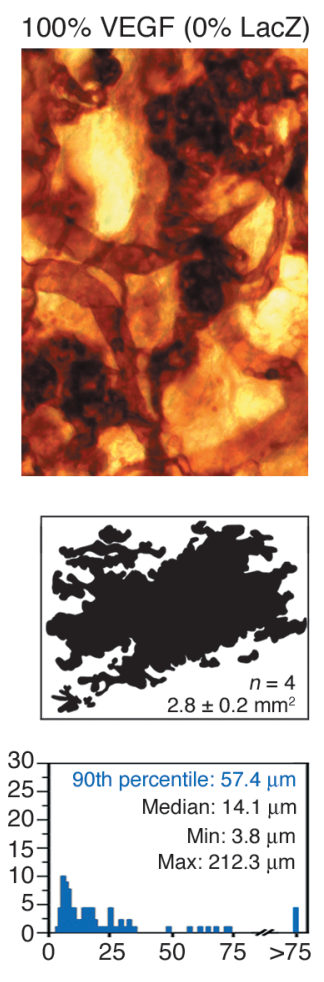

Figure 2

A reduction in the total amount of VEGF does not eliminate abnormal vascular growth. Delivery of a smaller proportion of VEGF myoblasts (less total VEGF) failed to prevent aberrant vascular growth at 28 days. (a) Lectin staining of the vasculature revealed focal growth of morphologically abnormal, bulbous vascular structures in ears implanted with a mixture consisting of 7-100\% VEGF myoblasts, with the remainder being control myoblasts. Vessels in control ears implanted with $100 \%$ LacZ myoblasts had a normal appearance. Myoblasts were stained for LacZ expression using the X-gal reaction (blue). (b) Implantation of increasing proportions of VEGF myoblasts resulted in larger areas $\left(\mathrm{mm}^{2}\right)$ of VEGF-induced vascular growth. Tracings of areas of vascular growth shown are from one representative sample per group; $n$ values and average area for each group are indicated. (c) Vessel diameter distribution measured at implantation sites of the control and VEGF myoblast dilutions shown in a. Implantation of different dilutions of the heterogeneous VEGF myoblast population invariably led to growth of vessels with similarly large and heterogeneous diameters. Min, minimum; Max, maximum. Scale bars: $50 \mu \mathrm{m}(\mathbf{a})$ and $300 \mu \mathrm{m}$ (b). 
extensive remodeling and were markedly enlarged by days 4-7 (Figure 1e). By 28 days, the implantation site was filled with bulbous vascular structures resembling glomeruloid bodies $(18,19)$ and small bundles of capillary-sized vessels (Figure 1f). These vascular structures were immunoreactive for the endothelial markers PECAM-1 and VE-cadherin (not shown). The neovascular growth promoted by VEGF became increasingly abnormal over time. By days 43-64, large blood-filled growths resembling hemangiomas (31) developed in all VEGF myoblast-implanted ears, but not in contralateral control ears (Figure 1, g and h). These hemangiomas were similar to vascular growths previously reported after VEGF myoblast implantation into other tissues $(15,16)$.

These effects of VEGF were highly localized. Vessels leading into the implantation site became morphologically abnormal immediately upon reaching the zone of VEGF-producing myoblasts, resulting in a sharp border between normal and affected vessels corresponding to the border of the transgenic muscle region (Figure 1, e and i). Given previously published results showing that after fusion of VEGF-secreting myoblasts into myofibers VEGF protein remains tightly localized around the secreting fibers in vivo (32), the boundary between normal and affected vessels likely correlates to a steep concentration gradient of VEGF. Within the same ear, vessels outside of the myoblast implantation site were normal (not shown). In addition, no increase in serum VEGF levels was observed after implantation of VEGF myoblasts at 18 hours or 7,14 , or 28 days ( $n=3$ per time point), compared with that of untreated mice $(n=2$, not shown).

The total level of VEGF does not determine vessel morphology. Next, we examined if the severity of the abnormality of VEGF-induced angiogenesis corresponded to the total amount of VEGF produced at the implantation site. We tested if we could correct the abnormal phenotype by reducing the number of VEGF myoblasts by diluting them with control myoblasts to keep the total cell number constant. Remarkably, even when only $7 \%$ of the total implanted myoblasts were VEGF myoblasts, small focal regions of aberrant, bulbous vascular structures typical of ears implanted with $100 \%$ VEGF myoblasts were present at 28 days (Figure $2 \mathrm{a}$ ), as were regions of increased capillaries with relatively normal vessel morphology (not shown). When ears were implanted with $15 \%$ or $30 \%$ VEGF myoblasts, abnormal vascular structures were also observed. Differences in the percentage of VEGF-producing myoblasts only affected the magnitude of the affected area. The total affected area, defined as the sum of areas $\left(\mathrm{mm}^{2}\right)$ where vascular growth was clearly observed (see Methods), correlated with the percentage of VEGF myoblasts implanted (Figure 2b; $r^{2}=0.72$ between ears implanted with 7-100\% VEGF myoblasts, $P<0.001)$. Thus, severely abnormal vessels, including the bulbous, glomeruloid vascular structures, were found in all ears that received cells from the heterogeneous polyclonal VEGF-expressing myoblast population irrespective of how few of these cells were implanted. Only the size of the area exhibiting vascular growth decreased with lower percentages of implanted VEGF myoblasts.

We quantified the morphological effect of VEGF delivery in each percentage group by obtaining random measurements of vessel diameters within affected areas (Figure 2c). The vessels in ears that received no VEGF (control myoblasts) were uniformly small in caliber, reflecting the prevalence of capillaries at the implantation site. Vessel diameters in ears receiving 7\%, 15\%, 30\% or $100 \%$ VEGF myoblasts were all significantly larger than those in the control myoblast ears $(P<0.0001$ for each group compared with control). Also, vessel diameter distributions were similarly aberrant among all the groups receiving from $7 \%$ to $100 \%$ VEGF myoblasts ( $P$ values ranged from 0.31 to 0.98 for the comparisons of each group with the others). In fact, vessel diameters within affected areas of ears implanted with $7 \%$ and $100 \%$ VEGF myoblasts were essentially identical $(P=0.98)$. Moreover, vessel diameters in ears implanted with VEGF-expressing myoblasts were heterogeneous compared with control myoblast ears, even when only $7 \%$ of the implanted myoblasts produced VEGF (Figure 2c). These VEGF-induced alterations in vessel diameter were due to the presence of aberrant, larger-caliber vascular structures. These results demonstrate that the formation of heterogeneous blood vessels of severely abnormal morphology was not prevented by reducing the total amount of VEGF delivered.

Microenvironmental VEGF dosage determines a threshold between normal and aberrant vascular growth. We next examined if uniformly lowering the amount of VEGF produced per cell might normalize vascular growth.

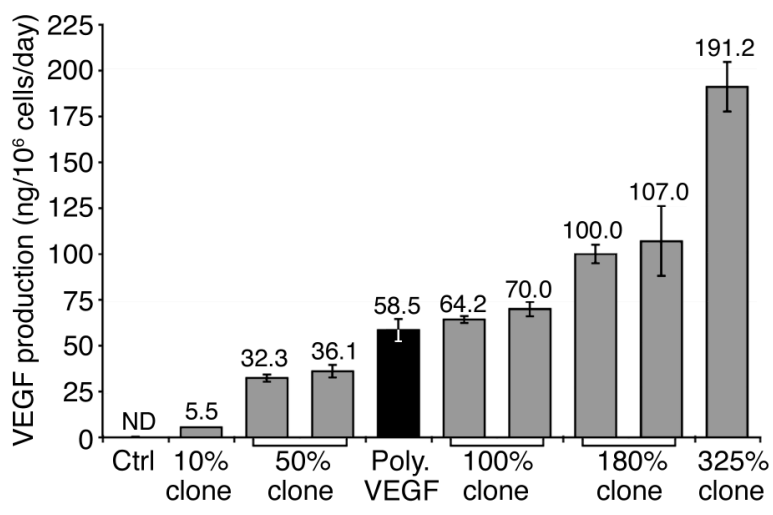

\section{Figure 3}

Uniform VEGF production across a broad range of levels by myoblast clones. Clones were selected that ranged in VEGF protein secretion levels from $10 \%$ to $325 \%$ of the average of the parent VEGF myoblast population; these are designated " $10 \%$ clone" and " $325 \%$ clone," for example. Actual VEGF production is indicated above the bar for each population (as ng/ $10^{6}$ cells/day). Error bars indicate the SEM of three replicate measurements per clone or population. Ctrl, LacZ-expressing control myoblasts; Poly. VEGF, original polyclonal VEGF myoblast population from which the clones were derived; ND, not detectable. 
a

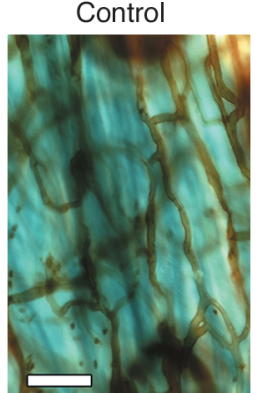

b

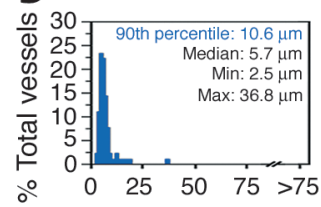

c

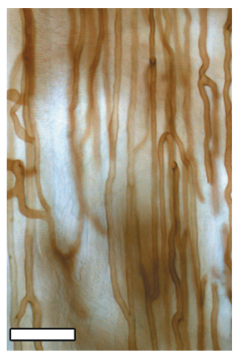

$10 \%$ clone
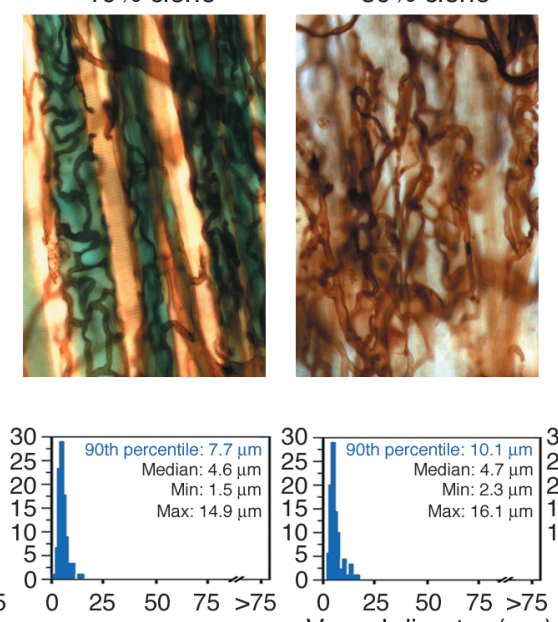

Vessel dimeter $(\mu \mathrm{m})$

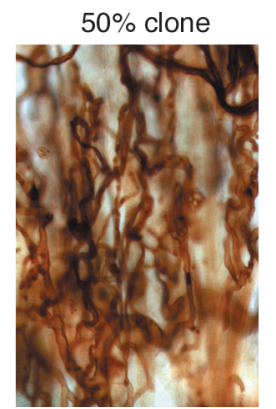

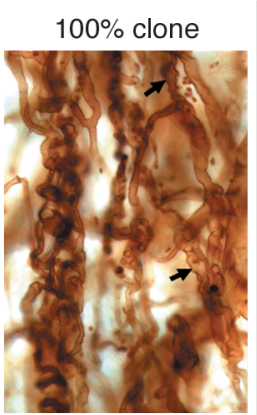
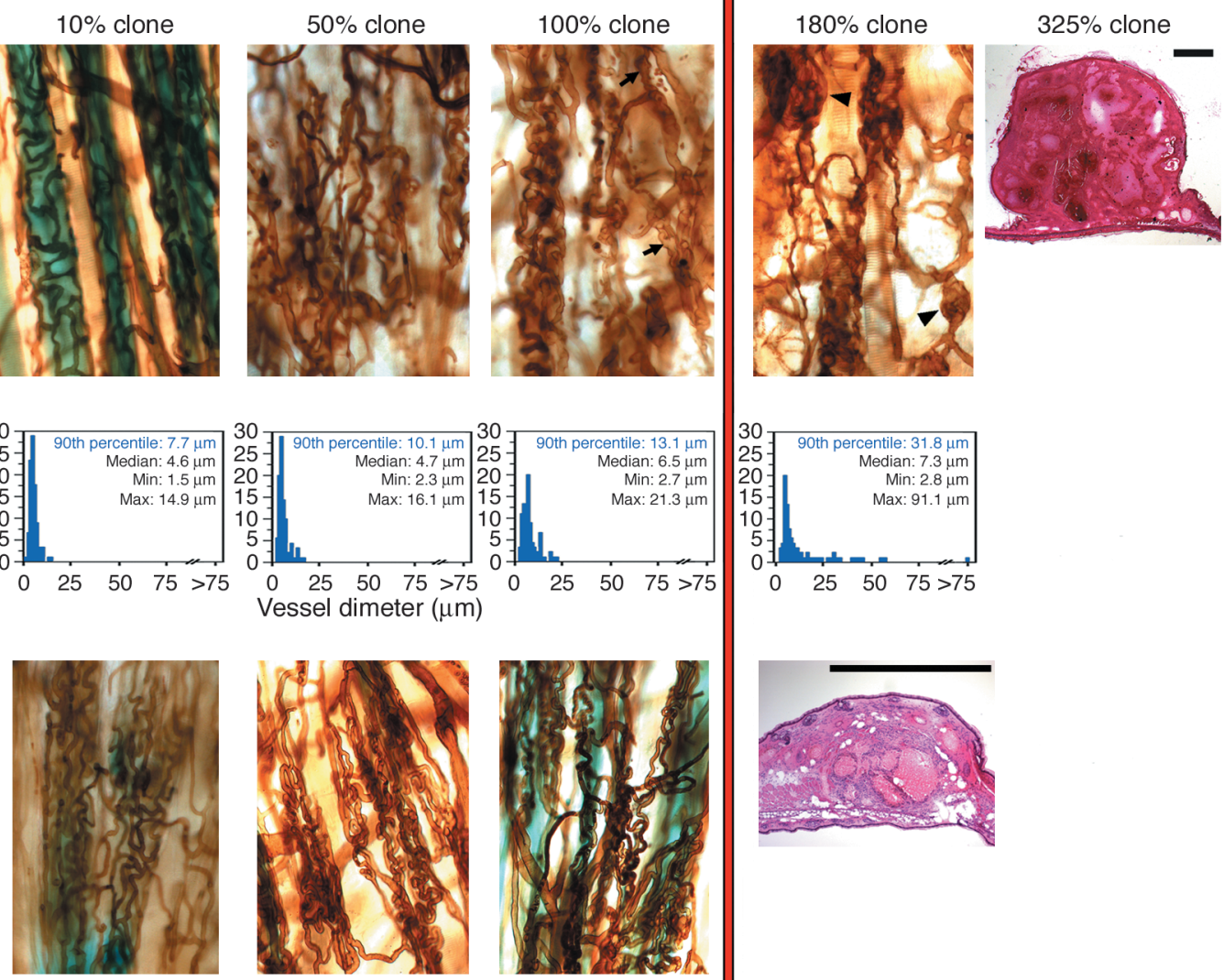

Capillaries
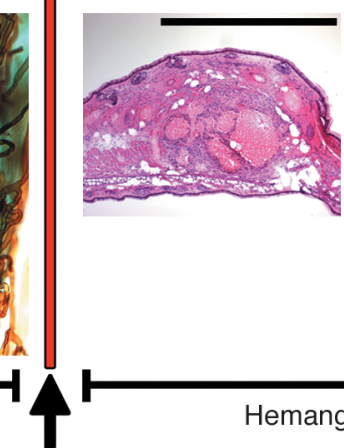

Hemangiomas

\section{Figure 4}

The microenvironmental level of VEGF produced in muscle determines a threshold between the growth of normal capillaries and hemangiomas. (a) Morphology of vessels at sites of implanted LacZ control cells (Control) and of different VEGF clones at 4 weeks $(n=3-7$ per group). Clones $10 \%, 50 \%$, and $100 \%$ produced small-caliber vessels resembling capillaries. Although vessels at sites implanted with $100 \%$ clones were uneven in diameter in some regions (arrows), no abnormal bulbous structures were observed in these ears. Aberrant bulbous vascular structures were present in ears implanted with the $180 \%$ clone (arrowheads). Large hemangiomas developed in ears implanted with the $325 \%$ clone by 4 weeks (H\&E stain). (b) The corresponding distributions of vessel diameters at implantation sites are shown below each panel. (c) Morphologies of vessels at sites implanted with the same clones at 2.5-3.5 months after implantation. The capillaries induced by the $10 \%, 50 \%$, and $100 \%$ clones persisted and did not develop any abnormalities, whereas hemangiomas grew in ears implanted with the $180 \%$ clone after 2.5 months (H\&E stain). The vertical red line and the arrow at the bottom indicate the threshold between the induction of normal capillaries and hemangiomas at a VEGF level between that produced by the $100 \%$ and $180 \%$ clones. Scale bars: $50 \mu \mathrm{m}$ (lectin panels, far right in a and c), $1 \mathrm{~mm}(\mathrm{H} \& \mathrm{E}$ panel in a), and $0.5 \mathrm{~mm}(\mathrm{H} \& \mathrm{E}$ panel in c).

This was achieved by isolation of clones of VEGF-producing myoblasts from the polyclonal parent population. Different cells in the transduced population expressed different VEGF levels depending on the number of retroviral copies that integrated and on the general transcriptional activity resulting from the chromatin organization around the random integration site. Single cells were isolated using a cell sorter and expanded in culture to produce monoclonal populations. Clones were selected that produced homogeneous amounts of VEGF ranging from approximately 10\% (5.5 $\mathrm{ng} / 10^{6}$ cells/day) to $325 \%$ ( $191.2 \mathrm{ng} / 10^{6}$ cells/day) of the average level produced by the polyclonal VEGF myoblasts $\left(58.5 \mathrm{ng} / 10^{6} \mathrm{cells} /\right.$ day $)$ in culture; these were designated, for example, "10\% clone" or " $325 \%$ clone" according to their VEGF production (Figure 3).
In contrast to the outcome after implantation of smaller proportions of the polyclonal VEGF population (see preceding section), the resultant vasculature after implantation of clones was uniform for each clone and the morphology correlated strictly with the amount of VEGF produced per clone. Vascular morphology was examined at 28 days after implantation. Low levels of VEGF production introduced by the $10 \%$ VEGF clone (producing $\sim 5 \mathrm{ng} / 10^{6}$ cells/day) resulted in growth of vessels that were morphologically normal and homogeneous in diameter (Figure 4a), resembling capillaries found in untreated or LacZ-implanted (control) ears. These vessels were distributed throughout the implantation site, were localized to VEGF-producing muscle fibers (identified by positive X-gal staining; Figure 4a), and were 


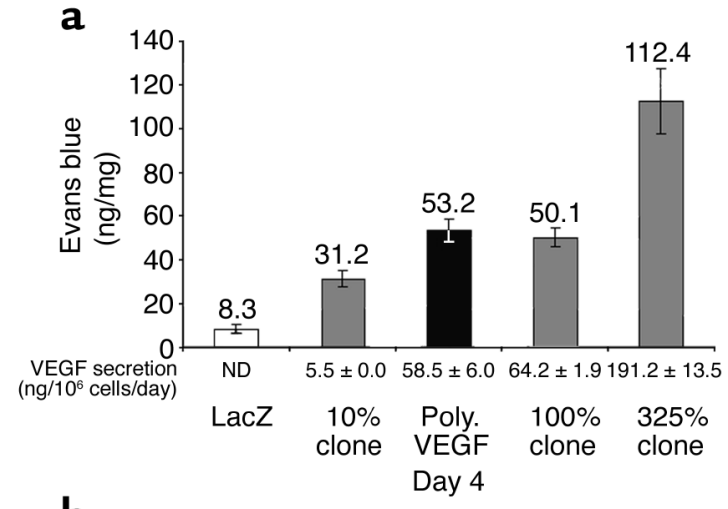

b

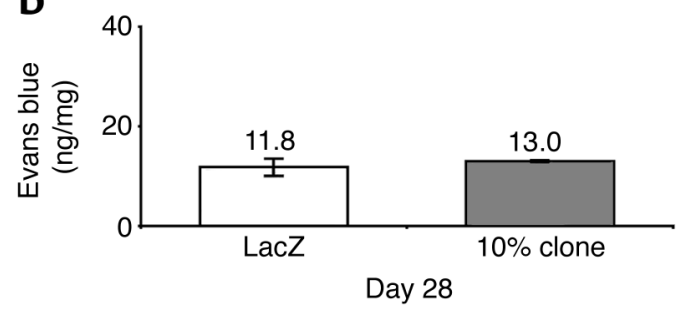

Figure 5

Plasma leakage correlates with the total amount of VEGF produced and is transient. (a) Evans blue extravasation at sites of implanted LacZ control, polyclonal VEGF (Poly. VEGF), and 10\%, $100 \%$, or $325 \%$ clone myoblasts at 4 days after implantation. ND, not detected. (b) Evans blue extravasation in ears implanted with LacZ control and $10 \%$ clone at 28 days after implantation. Leakage at this time point had returned to the baseline level. Values are mean $\pm \operatorname{SEM}(n=3-6$ per group $)$.

slightly smaller in diameter than capillaries found in control ears $(P=0.001$; Figure $4 \mathrm{~b})$.

Ears implanted with the $50 \%$ and $100 \%$ clones (producing $\sim 35$ and $\sim 70 \mathrm{ng} / 10^{6}$ cells/day, respectively) also displayed abundant capillary-like vessels throughout the implantation site (Figure 4a). Notably, although vessels at sites implanted with $100 \%$ clones were uneven in diameter in some regions (arrows in Figure $4 \mathrm{a}$ ), the abnormal bulbous structures observed in ears implanted with polyclonal VEGF myoblasts were never found (compare Figures $1 \mathrm{f}$ and 4a). This difference was clearly evident even though the two myoblast populations (polyclonal and clonal) secreted similar average amounts of VEGF in culture and the same number of cells had been implanted. Thus, although equivalent total amounts of VEGF were delivered, vessel diameters were statistically smaller $(P<0.0001)$ and were uniform (rather than heterogeneous) in ears implanted with $100 \%$ clones compared with in ears implanted with polyclonal VEGF myoblasts. Diameters of vessels induced by the $10 \%, 50 \%$, and $100 \%$ clones progressively increased with higher doses of VEGF production. In fact, the 90 th percentile of the diameter distribution increased from $7.7 \mu \mathrm{m}$ to $10.1 \mu \mathrm{m}$ and $13.1 \mu \mathrm{m}$, respectively (Figure $4 \mathrm{~b}$ ), and the diameters of vessels induced by $100 \%$ clones were statistically larger than those induced by either the $10 \%$ or
$50 \%$ clones $(P<0.0001)$. Thus, low to medium microenvironmental levels of VEGF production resulted in abundant new capillary-like vessels, without deleterious growth of abnormal bulbous vascular structures or hemangiomas.

In contrast, ears implanted with $180 \%$ clones (producing $\sim 100 \mathrm{ng} / 10^{6}$ cells/day) were notable for the appearance of aberrant bulbous structures (arrowheads in Figure 4a). Vessel diameters in these ears were larger and more heterogeneous than those in control ears; the 90th percentile of vessel diameter distribution was threefold higher in ears implanted with the $180 \%$ VEGF clone $(31.8 \mu \mathrm{m})$ than in control myoblast ears $(10.6 \mu \mathrm{m} ; P<0.001)$. Even more striking, the $325 \%$ clone (producing $\sim 190 \mathrm{ng} / 10^{6}$ cells/ day) promoted accelerated growth of large hemangiomas at 28 days (Figure 4a), similar in size to hemangiomas found in ears implanted with the polyclonal VEGF myoblasts at much longer time points (43-64 days; Figure 1, g and h).

Angiogenesis can be quantitatively assessed using measurements such as vessel length density; that is, the total length of vessels in a given area independent of vessel diameter or number (14). We confirmed that low levels of VEGF production resulted in a measurable increase in vascularity over controls by determining vessel length density in ears implanted with the $10 \%$ clone and in ears implanted with control myoblasts. The $10 \%$ clone induced a $56 \%$ increase in vessel length density $\left(87.0 \pm 2.9 \mathrm{~mm} / \mathrm{mm}^{2}\right)$ over that of controls $\left(55.7 \pm 3.8 \mathrm{~mm} / \mathrm{mm}^{2} ; P<0.005\right)$ at 4 weeks. Thus, a significant increase in angiogenesis was observed even with the $10 \%$ clone, which produced a very low level of VEGF $\left(\sim 5 \mathrm{ng} / 10^{6}\right.$ cells/day), and the newly formed vessels were normal in appearance.

Given that myoblast-mediated gene expression is stable over time and a lower VEGF level might take longer to induce an abnormal phenotype, we followed the evolution of the vessels induced by different VEGF levels over the long term (Figure 4c). The $10 \%, 50 \%$, and $100 \%$ clones all yielded an increased number of capillaries of homogenous size relative to neighboring nonimplanted regions and control myoblast-implanted ears at 2.5 months $(50 \%$ and $100 \%$ clones) and 3.5 months (10\% clone) after implantation, identical to those observed at 28 days. The bulbous structures induced by the $180 \%$ clone, however, enlarged, and by 2.5 months had evolved into hemangiomas, as with the $325 \%$ clone at 28 days. These results show that manipulation of microenvironmental VEGF dosage uncovered a wide range of expression levels that yielded homogeneous normal capillary-like vessels despite constitutive long-term production. The levels ranged from about $5 \mathrm{ng} / 10^{6}$ cells/day to at least $70 \mathrm{ng} / 10^{6}$ cells/day in culture, as the actual VEGF concentrations in vivo are difficult to assess because they are highly localized. Furthermore, these new normal vessels remained stable for at least several months, showing no signs of regres- 
sion or abnormal development. On the other hand, at VEGF levels between 70 and $100 \mathrm{ng} / 10^{6}$ cells/day and above, aberrant vasculature was induced that consistently evolved into continuously growing hemangiomas. Therefore, a threshold VEGF level appears to exist that separates the growth of capillary-like vessels from that of hemangiomas, with no stable intermediate abnormal structures.

Finally, we verified that these effects on vessel morphology were not specific to the parent myoblast population from which the clones were derived. New clones were isolated from a second heterogeneous population of myoblasts expressing recombinant VEGF $_{164}$ (called VEGF* here). Myoblasts derived from one VEGF* clone expressing low levels of VEGF (18 ng/ $10^{6}$ cells/day) and six clones expressing high levels (>100 ng/ $10^{6}$ cells/day) were implanted into the ears of mice and vessel morphologies were examined 28 days later. The new clone with low expression of VEGF also induced growth of homogeneous normal capillaries, while the clones with high expression of VEGF promoted heterogeneous, abnormal vessel growth $(n=2-9$ mice per clone, data not shown), confirming the studies shown in Figure 4. These findings are therefore reproducible across different myoblast populations.

Plasma leakage is transient and depends on the total amount of VEGF. Because VEGF induces both vascular growth and permeability, we determined if the morphologically distinct vessels induced by the VEGF clones also differed in function, assessed by the amount of plasma leakage. Evans blue extravasation $(14,25)$ was measured at myoblast implantation sites at 4 days, a time point chosen because plasma leakage induced by the polyclonal VEGF myoblast population in the ear peaks at 4 days (N. Glazer et al., manuscript submitted for publication). The amount of extravasated Evans blue correlated directly with the average amount of VEGF produced either by the myoblast clones or by the polyclonal VEGF population at this early time point (Figure 5a; $r^{2}=0.96, P<0.005$ ). Moreover, we examined if the morphologically normal vessels induced by the $10 \%$ clone were still leaky at a later time point (Figure 5b). At 28 days, there was no difference in Evans blue extravasation between ears implanted with the $10 \%$ clone and control ears. This shows that low levels of VEGF delivered constitutively over the long term resulted in increased numbers of stable vessels that were not only morphologically normal but also nonleaky.

Aberrant vascular growth results from "bot-spots" of bigh microenvironmental VEGF levels. We hypothesized that the abnormal vascular growth observed with VEGF gene delivery is due to high microenvironmental concentrations of VEGF introduced by cells with high expression within the polyclonal VEGF-secreting population and that this phenotype dominates the angiogenesis induced by cells with low and medium expression. To test this hypothesis, we mixed equal numbers of ten VEGF clones that ranged $~ 33$-fold in VEGF production, implanted them into the ear, and examined vessel morphologies at 28 days. Morphologies of the resulting vessels were similar to those observed after implantation of the original polyclonal, heterogeneous VEGF population (data not shown). Vessel diameters at implantation sites of the mixture of clones were statistically similar $(P=0.25)$ to those at implantation sites of polyclonal VEGF myoblasts, and the sites contained hemangiomas. These results show that the effects observed with the original polyclonal VEGF population could be recreated by mixing clonal populations secreting a broad range of VEGF levels.

To understand the contribution of cells with high expression of VEGF to the aberrant vascular growth induced by polyclonal VEGF myoblasts, we used myoblasts derived from the clone (325\%) with the highest expression and diluted them with control myoblasts, keeping the total cell number constant. Implantation of only $7 \%$ or $30 \%$ of these myoblasts with high expression of VEGF resulted in focal regions containing large vas-
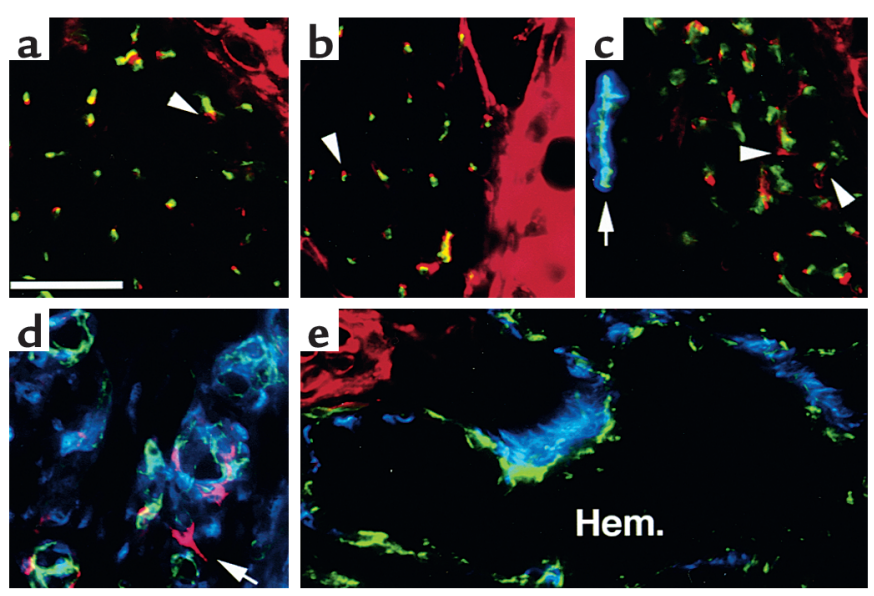

\section{Figure 6}

Phenotypically different mural cells are associated with vessels induced by different VEGF levels. (a-e) Frozen sections of normal ears (a) and ears implanted with control (LacZ) myoblasts (b), $10 \%$ clone (c), or $180 \%$ clone (d and $\mathbf{e}$ ) were immunostained for the endothelial marker PECAM-1 (in green) and the mural cell markers NG2 (in red) and $\alpha$-SMA (in blue) 4 weeks after implantation. Capillary-like vessels induced by VEGF levels below the threshold (c) were completely covered with normal pericytes (NG2-positive/ $\alpha$-SMA-negative perivascular cells; arrowheads in $\mathbf{a}-\mathbf{c}$ ), similar to those on pre-existing capillaries of normal muscle (a) and muscle implanted with control myoblasts (b), whereas $\alpha$-SMA is normally expressed by mural cells on arteries and veins (arrow in $\mathbf{c}$ ). Aberrant structures induced by VEGF levels above the threshold ( $\mathbf{d}$ and $\mathbf{e}$ ), however, possessed few NG2-positive/ $\alpha$-SMA-negative pericytes, which sometimes appeared only loosely associated with the endothelium (arrow in d) and were replaced by NG2-negative/ $\alpha$-SMA-positive cells. This phenotypic and morphologic difference in mural cell coverage correlated with the progressive growth of abnormal bulbous vessels into hemangiomas (Hem. in e). Scale bar: $50 \mu \mathrm{m}$. 

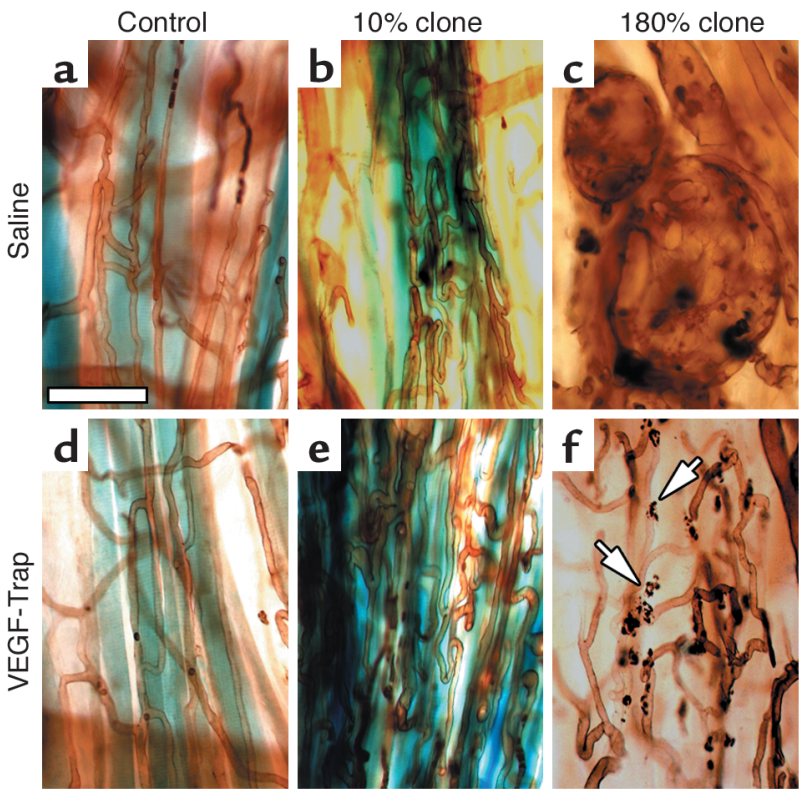

Figure 7

Normal capillaries induced by low VEGF levels are resistant to VEGF deprivation. (a-f) Mice were implanted with control myoblasts (a and $\mathbf{d}$ ), $10 \%$ clone (b and $\mathbf{e}$ ), or $180 \%$ clone (c and $\mathbf{f}$ ) and after 24 days were treated with intraperitoneal injections of saline $(\mathbf{a}-\mathbf{c})$ or VEGF-Trap 1 R2 (VEGF-Trap; d-f) for 4 days. The morphology of the induced vessels was analyzed by lectin staining after 28 days. Potent VEGF blockade by VEGF-TrapR1R2 had no effect on the stable new capillaries induced by low VEGF levels, whereas the aberrant vascular structures induced by a VEGF dose above the threshold regressed. Extravasated leukocytes are indicated by the arrows in $\mathbf{f}$. Saline treatment had no effect on any vessels. Scale bar: $50 \mu \mathrm{m}$.

cular sacs and hemangiomas (data not shown). Affected areas in these ears contained only vascular structures with very large vessel diameters (90th-percentile values of $174 \mu \mathrm{m}$; data not shown). This result demonstrates that abnormal vessel growth was induced even when as few as $7 \%$ of cells expressing VEGF at high levels were present. Heterogeneous VEGF production, such as that resulting from implantation of polyclonal VEGF myoblasts, caused abnormal vessel growth due to the presence of cells with high expression of VEGF. These results underscore the localized effects of VEGF concentration on angiogenesis and the importance of controlling its dosage on a microenvironmental scale.

Normal and aberrant vasculature is associated with phenotypically different mural cells. We determined whether mural cell coverage had a role in the behavior of vascular structures induced by VEGF produced in amounts above or below the threshold for the formation of aberrant blood vessels. Control myoblasts, $10 \%$ clone cells (below the threshold), or $180 \%$ clone cells (above the threshold) were implanted into ears and after 4 weeks the tissues were removed. Freshfrozen sections were immunostained for expression of PECAM-1 (endothelial cells), collagen IV (basal lamina), $\alpha$-SMA, and NG2 (mural cells). Pericytes were identified as perivascular cells that were closely associated with endothelial cells, had a characteristic morphology with thin processes running along the vessel and enveloping it in places, and were surrounded by basement membrane. Pericytes on normal capillaries in skeletal muscle were immunoreactive for the proteoglycan NG2 but not $\alpha$-SMA (arrowhead in Figure 6a), whereas mural cells on arteries and veins were immunoreactive for both NG2 and $\alpha$-SMA (arrow in Figure $6 c$ ). Pericytes were associated with all new capillary-like vessels induced by the implantation of the $10 \%$ clone (arrowheads in Figure $6 \mathrm{c}$ ), and these were similar to pericytes on capillaries in muscle implanted with control cells (arrowhead in Figure 6b). However, most pericytes on the aberrant bulbous structures induced by the $180 \%$ clone were NG2 negative/ $\alpha$-SMA positive (Figure 6 , $\mathrm{d}$ and $\mathrm{e}$ ). The few NG2-positive pericytes on these aberrant structures were loosely associated with endothelial cells and appeared to make imperfect cell-cell contacts (arrow in Figure 6d). Blood vessels in hemangiomas were covered exclusively with $\alpha$-SMApositive cells and had no NG2-positive/ $\alpha$-SMA-negative pericytes (Figure 6e). Thus, the formation of aberrant blood vessels, which were destined to become hemangiomas, was accompanied by the replacement of NG2-positive/ $\alpha$-SMA-negative pericytes with NG2-negative/ $\alpha$-SMA-positive cells.

Normal capillaries induced by low VEGF levels become VEGF independent. To be therapeutically useful, new vessels induced by an angiogenic treatment must be stable and persist after cessation of the treatment. We investigated the VEGF dependence of normal and aberrant blood vessels induced by VEGF below and above the threshold level by treating mice with VEGF-

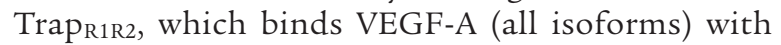
high affinity and prevents its binding to cellular VEGF receptors, thereby blocking its action in vivo (30). Ears were injected with control, $10 \%$ clone, or $180 \%$ clone myoblasts and mice were treated with VEGF-Trap $_{\text {R1R2 }}$ or saline for 4 days before analysis of vascular morphology by lectin staining. The initial angiogenic vessels induced 1 week after implantation of clones with either low or high expression were completely abolished by VEGF-Trapr1R2 treatment, but were unaffected by saline treatment (data not shown). In contrast, at 4 weeks after implantation, the normal capillaries induced by low VEGF levels had become resistant to VEGF deprivation (Figure 7e). However, strikingly, the aberrant structures induced by VEGF levels above the threshold were still completely eliminated by VEGF-Trap ${ }_{R 1 R 2}$ treatment (Figure 7f). Extravasated leukocytes, recognized by their peroxidasepositive granules (arrows in Figure 7f), were abundant near regressing aberrant vessels and may have participated in clearing cellular debris. These results show that the morphologically normal vessels induced by low microenvironmental VEGF levels become stabilized as defined by VEGF independence by 3.5 weeks after myoblast implantation (as the VEGF-Trapr1R2 
treatment was started at day 24), whereas the aberrant vessels induced by high levels of VEGF remain dependent on continued VEGF supply.

\section{Discussion}

Despite its promise for treating ischemic diseases (for a review, see ref. 33), VEGF can induce the growth of aberrant blood vessels and hemangiomas (5, 15-19), and this could limit its therapeutic utility. In the present study we addressed the question of whether VEGF can be administered in a way that favors normal angiogenesis and, if so, what the optimal conditions for achieving this goal are. Our experiments provided evidence for three new findings: (a) constitutive longterm VEGF gene expression in adult muscle can induce stable, normal angiogenesis when the dosage is precisely controlled; (b) VEGF effects depend on microenvironmental, and not total, VEGF dosage, as averaging of different levels over an area of muscle does not occur; and (c) a discrete threshold in VEGF dosage exists below which normal, stable capillaries are induced and above which hemangioma growth occurs, with no stable intermediate phenotypes. When produced under optimal conditions, VEGF promoted growth of capillaries of uniform size that were not leaky at 28 days, were stable for at least 3.5 months, and were independent of continued VEGF expression. Implantation of even small numbers of myoblasts with high expression of VEGF resulted in growth of aberrant vascular structures, demonstrating that extremely localized levels of VEGF determine the morphology of the new vessels.

With homogeneous expression by clones, an increase in the dose up to about $70 \mathrm{ng} / 10^{6}$ cells/day was accompanied by a gradual increase in the diameter of the new vessels, but without the formation of pathological structures. At doses of about $100 \mathrm{ng} / 10^{6}$ cells/day and above, however, bulbous glomeruloid bodies appeared early and full-fledged hemangiomas invariably developed over time. This dosage interval (between 70 and $100 \mathrm{ng} / 10^{6}$ cells/day) appears to represent a threshold level between VEGF-induced angiogenesis and "hemangioma-genesis" (34). A possible explanation is that such threshold could be determined by the balance between the local concentrations of proangiogenic and vessel stabilizing factors. Below the threshold, sufficient amounts of endogenous vessel maturation factors, either preexisting or induced by the delivered VEGF itself, may be present to produce normal vessel assembly and stabilization. Above the threshold, VEGF production presumably tips the balance toward exaggerated proangiogenic activity and unregulated growth of aberrant blood vessels and hemangiomas. In support of this possibility, we found that the phenotype of mural cells associated with the different kinds of vessels differed dramatically. Pericytes with a phenotype resembling that of pericytes on normal muscle capillaries (NG2-positive/ $\alpha$-SMA-negative) were in fact scarce on aberrant structures induced by VEGF levels above the threshold and in their place were mural cells immunoreactive for $\alpha$-SMA. A similar switch in expression of $\alpha$-SMA by aberrant pericytes has been described recently in the abnormal vessels of the RIPTag2 genetic model of pancreatic islet tumors (35). Interestingly, this phenotypic transition correlated with tumor progression: no pericytes expressed $\alpha$-SMA on capillaries in normal pancreatic islets; some pericytes expressed $\alpha$-SMA on vessels in hyperplastic premalignant islets; and virtually all vessels in advanced cancers had $\alpha$-SMA-positive aberrant pericytes. Our results show that the appearance of mural cells with a similar phenotype on vessels induced by VEGF levels above the threshold correlates with aberrant vascular morphology, progressive growth to hemangiomas (Figure 4), and continued dependence on VEGF (Figure 7). On the other hand, the blood vessels induced by VEGF levels below the threshold displayed capillary-like morphology, were tightly associated with NG2-positive/ $\alpha$-SMA-negative pericytes, did not regress or change morphology over at least 3.5 months, and became independent of continued VEGF expression by 3.5 weeks; that is, they were truly stabilized. These observations are consistent with previous evidence for a paracrine/juxtacrine inhibition of endothelial cell proliferation by pericytes in vitro $(36,37)$ and during development $(38)$, pointing to a possible role of the balance between VEGFinduced angiogenesis and pericyte-mediated vascular maturation in determining the observed threshold between the induction of stable capillaries and unstable hemangiomas.

Upon injection into host muscle, most myoblasts die, while some proliferate and fuse either to pre-existing fibers or among themselves (26). In a clinical study of Duchenne muscular dystrophy, the number of donor nuclei stably participating in hybrid fibers was found to be about $10 \%$ of the injected myoblasts (39). In mice, the number of hybrid fibers remained constant from 5 days to 6 months after implantation (26) and quantification of reporter gene expression (LacZ) correlated tightly with the number of transgenic fibers (40). Although a determination of the precise amount of VEGF produced by myoblasts after integration in the injected muscle is impossible, we have recently shown that after myoblast fusion, VEGF protein remains tightly localized around the secreting fibers in vivo, creating a steep VEGF concentration gradient to which neighboring cells respond (32). The $\mathrm{VEGF}_{164}$ isoform delivered binds heparin $(41,42)$ and is likely to localize around each VEGF-producing cell. This concentration gradient would explain why the different vascular effects depend on microenvironmental, rather than total (or averaged), VEGF levels produced across a broad region of muscle.

The results presented here have clear implications for clinical applications of VEGF gene delivery aimed at promoting therapeutic angiogenesis. Currently 
utilized delivery methods, such as injection of constitutively expressing VEGF plasmids or adenovirus, carry an inherent potential for heterogeneous VEGF production within treated areas, depending for example on the number of plasmid copies or adenovirus particles taken up by individual muscle fibers. In light of the data we present here, simply controlling VEGF dose by altering the total amount of vector delivered will not regulate microenvironmental VEGF levels, and this may impose significant limits on the effective dose achieved. In fact, to be safe, even the foci of highest expression must be below the threshold level, which implies that most areas are likely to have much lower microenvironmental VEGF than they might safely achieve otherwise. Consequently, room for increased efficacy is wasted (43-45). The development and delivery of expression vectors that can be regulated and that can appropriately tailor levels of VEGF expression could be useful to circumvent this (46). Alternatively, controlledrelease polymers may provide a solution. These represent a diverse class of biomaterials that allow the homogeneous delivery of one or even two factors simultaneously $(47,48)$. The chemical properties of the polymer scaffold can be engineered to control precisely both the rate and the time over which a certain dose of recombinant protein is released in vivo, so that treatment strategies can be modified as new insights emerge about the basic biology of angiogenesis. Thus, this method of delivery potentially holds great promise for achieving control over the microenvironmental levels of angiogenic factors and their homogeneous distribution.

Growth of hemangiomas has not been a major issue in patient trials to date, mainly because methods of gene transfer have been relatively inefficient or have led to only short-term expression of VEGF; a hemangioma appearing in one patient treated with a VEGF-expressing plasmid disappeared as recombinant VEGF expression was lost over time (5). It should be noted that we found the range of VEGF produced from implanted cells that induce normal neovascularization without signs of aberrant growth to be quite wide, from approximately $5 \mathrm{ng} / 10^{6}$ cells/ day to at least $70 \mathrm{ng} / 10^{6}$ cells/day in culture. Also, such vessels correctly matured, stabilized, and were VEGF independent by 3.5 weeks, showing the potential for indefinite persistence after discontinuation of treatment. This bodes well for the achievement of a manageable therapeutic window in clinical applications. However, for successful treatment of a wide variety of patients, it would be useful to be able to take advantage of the full range of microenvironmental therapeutic VEGF levels within the safe window. This window will need to be determined in ischemic conditions and for different delivery methods, as the amounts of VEGF produced by myoblasts in vitro cannot be directly extrapolated to the microenvironmental concentrations achieved in vivo.
Angiogenesis is a complex process and, although coregulation of more than one factor is being actively investigated (20), our results demonstrate that when expressed in appropriate microenvironmental amounts, even the long-term delivery of a single factor, VEGF, can safely induce growth of stable vessels with normal morphology and function. While it is a technical challenge, the development of delivery methods that can control the level of VEGF delivered in a homogeneous fashion could significantly improve the efficacy and flexibility of VEGF-based therapeutic angiogenesis.

\section{Acknowledgments}

We thank Fabienne Baffert and Michael RochonDuck (University of California at San Francisco) for assistance with vessel measurements, and Georges von Degenfeld (Stanford University) for invaluable comments on the manuscript. We thank Regeneron Corporation for the VEGF-TrapR1R2 and Kevin Claffey (University of Connecticut, Farmington, Connecticut, USA) for the $\mathrm{VEGF}_{164} \mathrm{cDNA}$. This work was supported by a predoctoral fellowship from the Howard Hughes Medical Institute (C.R. Ozawa), funds from NIH grants HL-59157 and HL-24136 (D.M. McDonald) and funds from NIH grants HL65572, HD-18179, AG-09521, and CA-59717 and the Baxter Foundation (H.M. Blau).

1. Ferrara, N. 2000. VEGF: an update on biological and therapeutic aspects. Curr. Opin. Biotechnol. 11:617-624.

2. Jones, N., Iljin, K., Dumont, D.J., and Alitalo, K. 2001. Tie receptors: new modulators of angiogenic and lymphangiogenic responses. Nat. Rev. Mol. Cell. Biol. 2:257-267.

3. Yancopoulos, G.D., Davis, S., Gale, N.W., Rudge, J.S., Wiegand, S.J., and Holash, J. 2000. Vascular-specific growth factors and blood vessel formation. Nature. 407:242-248.

4. Carmeliet, P., and Jain, R.K. 2000. Angiogenesis in cancer and other diseases. Nature. 407:249-257.

5. Isner, J.M., et al. 1996. Clinical evidence of angiogenesis after arterial gene transfer of phVEGF165 in patient with ischaemic limb. Lancet. 348:370-374.

6. Mack, C.A., et al. 1998. Biologic bypass with the use of adenovirus-mediated gene transfer of the complementary deoxyribonucleic acid for vascular endothelial growth factor 121 improves myocardial perfusion and function in the ischemic porcine heart. J. Thorac. Cardiovasc. Surg. 115:168-176

7. Magovern, C.J., et al. 1997. Regional angiogenesis induced in nonischemic tissue by an adenoviral vector expressing vascular endothelial growth factor. Hum. Gene Ther. 8:215-227.

8. Muhlhauser, J., et al. 1995. VEGF165 expressed by a replication-deficient recombinant adenovirus vector induces angiogenesis in vivo. Circ. Res. 77:1077-1086.

9. Takeshita, S., et al. 1996. Gene transfer of naked DNA encoding for three isoforms of vascular endothelial growth factor stimulates collateral development in vivo. Lab. Invest. 75:487-501.

10. Tsurumi, Y., et al. 1996. Direct intramuscular gene transfer of naked DNA encoding vascular endothelial growth factor augments collateral development and tissue perfusion. Circulation. 94:3281-3290.

11. Drake, C.J., and Little, C.D. 1995. Exogenous vascular endothelial growth factor induces malformed and hyperfused vessels during embryonic neovascularization. Proc. Natl. Acad. Sci. U. S. A. 92:7657-7661.

12. Ash, J.D., and Overbeek, P.A. 2000. Lens-specific VEGF-A expression induces angioblast migration and proliferation and stimulates angiogenic remodeling. Dev. Biol. 223:383-398.

13. Dor, Y., et al. 2002. Conditional switching of VEGF provides new insights into adult neovascularization and pro-angiogenic therapy. EMBOJ. 21:1939-1947.

14. Thurston, G., et al. 1999. Leakage-resistant blood vessels in mice transgenically overexpressing angiopoietin-1. Science. 286:2511-2514.

15. Springer, M.L., Chen, A.S., Kraft, P.E., Bednarski, M., and Blau, H.M. 
1998. VEGF gene delivery to muscle: potential role for vasculogenesis in adults. Mol. Cell. 2:549-558.

16. Lee, R.J., et al. 2000. VEGF gene delivery to myocardium: deleterious effects of unregulated expression. Circulation. 102:898-901.

17. Schwarz, E.R., et al. 2000. Evaluation of the effects of intramyocardial injection of DNA expressing vascular endothelial growth factor (VEGF) in a myocardial infarction model in the rat-angiogenesis and angioma formation. J. Am. Coll. Cardiol. 35:1323-1330.

18. Pettersson, A., et al. 2000. Heterogeneity of the angiogenic response induced in different normal adult tissues by vascular permeability factor/vascular endothelial growth factor. Lab. Invest. 80:99-115.

19. Sundberg, C., et al. 2001. Glomeruloid microvascular proliferation follows adenoviral vascular permeability factor/vascular endothelial growth factor-164 gene delivery. Am. J. Pathol. 158:1145-1160.

20. Blau, H.M., and Banfi, A. 2001. The well-tempered vessel. Nat. Med. 7:532-534.

21. Carmeliet, P., et al. 1996. Abnormal blood vessel development and lethality in embryos lacking a single VEGF allele. Nature. 380:435-439.

22. Ferrara, N., et al. 1996. Heterozygous embryonic lethality induced by targeted inactivation of the VEGF gene. Nature. 380:439-442.

23. Miquerol, L., Langille, B.L., and Nagy, A. 2000. Embryonic development is disrupted by modest increases in vascular endothelial growth factor gene expression. Development. 127:3941-3946.

24. Suri, C., et al. 1998. Increased vascularization in mice overexpressing angiopoietin-1. Science. 282:468-471.

25. Thurston, G., et al. 2000. Angiopoietin-1 protects the adult vasculature against plasma leakage. Nat. Med. 6:460-463.

26. Rando, T.A., and Blau, H.M. 1994. Primary mouse myoblast purification, characterization, and transplantation for cell-mediated gene therapy. J. Cell Biol. 125:1275-1287.

27. Springer, M.L., and Blau, H.M. 1997. High-efficiency retroviral infection of primary myoblasts. Somat. Cell Mol. Genet. 23:203-209.

28. Field, J., et al. 1988. Purification of a RAS-responsive adenylyl cyclase complex from Saccharomyces cerevisiae by use of an epitope addition method. Mol. Cell. Biol. 8:2159-2165.

29. Springer, M.L., Rando, T.A., and Blau, H.M. 1997. Gene delivery to muscle. In Curent protocols in human genetics. A.L. Boyle, editor. John Wiley \& Sons. New York, New York, USA. 13.4.1-13.4.19

30. Holash, J., et al. 2002. VEGF-Trap: a VEGF blocker with potent antitumor effects. Proc. Natl. Acad. Sci. U. S. A. 99:11393-11398.

31. Rosai, J. 1996. Soft tissues. In Ackerman's surgical pathology. Mosby-Year Book Inc. St. Louis, Missouri, USA. 2061-2064.

32. Springer, M.L., et al. 2003. Localized arteriole formation directly adjacent to the site of VEGF-Induced angiogenesis in muscle. Mol. Ther. 7:441-449.

33. Isner, J.M. 2002. Myocardial gene therapy. Nature. 415:234-239.
34. Carmeliet, P. 2000. VEGF gene therapy: stimulating angiogenesis or angioma-genesis? Nat. Med. 6:1102-1103.

35. Morikawa, S., et al. 2002. Abnormalities in pericytes on blood vessels and endothelial sprouts in tumors. Am. J. Pathol. 160:985-1000.

36. Hirschi, K.K., Rohovsky, S.A., Beck, L.H., Smith, S.R., and D'Amore, P.A. 1999. Endothelial cells modulate the proliferation of mural cell precursors via platelet-derived growth factor-BB and heterotypic cell contact. Circ. Res. 84:298-305.

37. Antonelli-Orlidge, A., Saunders, K.B., Smith, S.R., and D'Amore, P.A. 1989 An activated form of transforming growth factor beta is produced by cocultures of endothelial cells and pericytes. Proc. Natl. Acad. Sci. U. S. A. 86:4544-4548.

38. Hellstrom, M., et al. 2001. Lack of pericytes leads to endothelial hyperplasia and abnormal vascular morphogenesis. J. Cell Biol. 153:543-553.

39. Gussoni, E., Blau, H.M., and Kunkel, L.M. 1997. The fate of individual myoblasts after transplantation into muscles of DMD patients. Nat. Med. 3:970-977.

40. Rando, T.A., Pavlath, G.K., and Blau, H.M. 1995. The fate of myoblasts following transplantation into mature muscle. Exp. Cell Res. 220:383-389.

41. Houck, K.A., Leung, D.W., Rowland, A.M., Winer, J., and Ferrara, N. 1992. Dual regulation of vascular endothelial growth factor bioavailability by genetic and proteolytic mechanisms. J. Biol. Chem. 267:26031-26037.

42. Park, J.E., Keller, G.A., and Ferrara, N. 1993. The vascular endothelial growth factor (VEGF) isoforms: differential deposition into the subepithelial extracellular matrix and bioactivity of extracellular matrix-bound VEGF. Mol. Biol. Cell. 4:1317-1326.

43. Simons, M., et al. 2000. Clinical trials in coronary angiogenesis: issues, problems, consensus: An expert panel summary. Circulation. 102:E73-E86

44. Epstein, S.E., Fuchs, S., Zhou, Y.F., Baffour, R., and Kornowski, R. 2001. Therapeutic interventions for enhancing collateral development by administration of growth factors: basic principles, early results and potential hazards. Cardiovasc. Res. 49:532-542.

45. Isner, J.M., Vale, P.R., Symes, J.F., and Losordo, D.W. 2001. Assessment of risks associated with cardiovascular gene therapy in human subjects. Circ. Res. 89:389-400.

46. Ozawa, C.R., Springer, M.L., and Blau, H.M. 2000. A novel means of drug delivery: myoblast-mediated gene therapy and regulatable retroviral vectors. Annu. Rev. Pharmacol. Toxicol. 40:295-317.

47. Lutolf, M.P., et al. 2003. Synthetic matrix metalloproteinase-sensitive hydrogels for the conduction of tissue regeneration: engineering cellinvasion characteristics. Proc. Natl. Acad. Sci. U. S. A. 100:5413-5418.

48. Richardson, T.P., Peters, M.C., Ennett, A.B., and Mooney, D.J. 2001. Polymeric system for dual growth factor delivery. Nat. Biotechnol. 19:1029-1034. 